\title{
Dominating Cliques in Distance-Hereditary Graphs *
}

\author{
Feodor F. Dragan \\ Dept. of Math. and Cybern. Moldova State University \\ A. Mateevici str. 60 Chişinău 277009 Moldova
}

\begin{abstract}
A graph is distance-hereditary if and only if each cycle on five or more vertices has at least two crossing chords. We present linear time algorithms for the minimum $r$-dominating clique and maximum strict $r$-packing set problems on distance-hereditary graphs. Some related problems such as diameter, radius, central vertex, $r$-dominating by cliques and $r$-dominant clique are investigated too.
\end{abstract}

\section{Introduction}

A subset $D \subset V$ is a dominating set in graph $G=(V, E)$ iff for all vertices $v \in V \backslash D$ there is a vertex $u \in D$ with $u v \in E$. It is a dominating clique in $G$ iff $D$ is a dominating set in $G$ and a clique (i.e. for all $u, v \in D u v \in E$ ).

There are many papers investigating the problem of finding minimum dominating sets in graphs with (and without) additional requirements to the dominating sets. The problems are in general $N P$-complete. For more special graphs the situation is sometimes better (for a bibliography on domination cf. [12], for a recent survey on special graph classes cf. [4]).

Opposite to dominating set for a given graph $G$ a dominating clique does not necessarily exist. As was shown in [6] the problem whether a given graph has a dominating clique is $N P$-complete even for weakly chordal graphs (a graph $G$ is weakly chordal iff $G$ does not contain induced cycles $C_{k}$ of length $k \geq 5$ and no complements $\overline{C_{k}}$ of such cycles).

[14] and [15] investigate the dominating clique problem on strongly chordal and chordal graphs. A graph $G$ is chordal iff it does not contain any induced cycle $C_{k}$ of length $k \geq 4$. A graph $G$ is strongly chordal iff it is chordal and each cycle in $G$ of even length at least 6 has an odd chord (a chord $x_{i}, x_{j}$ in a cycle $C=\left(x_{1}, \ldots, x_{2 k}\right)$ of even length $2 k$ is an odd chord if the distance in $C$ between $x_{i}$ and $x_{j}$ is odd). Although for chordal graphs there is a simple criterion for the existence of dominating cliques the problem of finding a minimum (cardinality) dominating clique is $N P$-complete. In the case of strongly chordal graphs the last problem is already polynomial-time solvable.

*This work was partially supported by the VW-Stiftung Project No. I/69041 e-mail address: dragan@university.moldova.su 
Here we study the following generalized domination ( $r$-domination) problem: Let $\left(r\left(v_{1}\right), \ldots, r\left(v_{n}\right)\right)$ be a sequence of non-negative integers which is given together with the input graph. For any two vertices $u, v$ denote by $\operatorname{dist}(u, v)$ the length (i.e. number of edges) of a shortest path between $u$ and $v$ in $G$. A subset $D \subset V$ is an $r$-dominating set in $G$ iff for all $v \in V \backslash D$ there is a $u \in D$ with $\operatorname{dist}(u, v) \leq r(v)$. It is an $r$-dominating clique in $G$ iff $D$ is additionally a clique.2

[9] investigates the $r$-dominating clique problem on Helly, chordal and dually chordal graphs. For the definition of dually chordal graphs see [5]. The condition for the existence of dominating cliques known from [15] on chordal graphs is shown to be valid also in more general case of $r$-domination in Helly graphs and in chordal graphs. Again the problem of finding a minimum $r$-dominating clique is $N P$-complete in Helly graphs and is linear-time solvable in dually chordal graphs (a superclass of strongly chordal graphs and subclass of Helly graphs).

In this paper we investigate the $r$-dominating clique problem on distancehereditary graphs. A distance-hereditary graph is a connected graph in which every induced path is isometric. That is, the distance of any two vertices in an induced path equals their distance in the graph. These graphs were introduced by E.HowORKA [13], who gave first characterizations of distance-hereditary graphs. For instance, a connected graph $G$ is distance-hereditary if and only if every circuit in $G$ of length at least 5 has a pair of chords that cross each other. Evidently every distance-hereditary graph is weakly chordal. We show that the condition for the existence of $r$-dominating cliques known from [9] on Helly graphs and chordal graphs is still valid in the case of distance-hereditary graphs. Also we give efficient algorithms for the minimum $r$-dominating clique problem and some related problems (such as diameter, radius, central vertex, $r$-dominating by cliques and $r$-dominant clique) on these graphs.

As we already mentioned the minimum $r$-dominating clique problem is $N P$ complete on chordal graphs and linear-time solvable in dually chordal graphs. Opposite to this problem the problems of $\mathrm{r}$-dominating by cliques and $\mathrm{r}$-dominant clique are $N P$-complete on dually chordal graphs and polynomial time solvable in chordal graphs (see [9]). So, the obtained results show that the distancehereditary graphs possess advantages of both chordal and dually chordal classes of graphs.

\section{Terminology and Basic Properties}

We shall consider finite, simple loopless, undirected and connected graph $G=$ $(V, E)$, where $V=\left\{v_{1}, \ldots, v_{n}\right\}$ is the vertex set and $E$ is the edge set of $G$, and we shall use more-or-less standard terminology from graph theory [10].

Let $v$ be a vertex of $G$. We denote the neighborhood of $v$, consisting of all vertices adjacent to $v$, by $N(v)$, and the closed neighborhood of $v$, the set $N(v) \cup\{v\}$, by $N[v]$. The $k$-th neighborhood of $v$, denoted by $N^{k}(v)$, is defined as the set of all vertices of distance $k$ to $v$, that is, $N^{k}(v)=\{u \in V: \operatorname{dist}(u, v)=k\}$. The disk centered at $v$ with radius $k$ is the set of all vertices having distance 
at most $k$ to $v: D^{k}(v)=\{u \in V: \operatorname{dist}(u, v) \leq k\}$. Let also $N^{k}(v, u)=$ $N^{k}(v) \cap N^{d i s t(u, v)-k}(u)$.

A vertex $v$ of $G$ is a leaf if $|N(v)|=1$. Two vertices $v$ and $u$ are twins if they have the same neighborhood $(N(v)=N(u))$ or the same closed neighborhood $(N[v]=N[u])$. True twins are adjacent, false twins are not. We denote with $<S>$ the subgraph of $G$ induced by the vertices of $S \subset V$. A cograph is a graph that does not contain any induced path of length three.

Several interesting characterizations of distance-hereditary graphs in terms of existence of particular kinds of vertices (leaves, twins) and in terms of metric and neighborhood properties, and forbidden configurations were provided by BANDELT and Mulder [2], and by D'ATri and Moscarini [8]. Some algorithmic aspects are considered in [11] and [8]. The following propositions list the basic information on distance-hereditary graphs that is needed in the sequel.

Proposition $1([2],[8])$ For a graph $G$ the following conditions are equivalent:

(1) $G$ is distance-hereditary.

(2) The house, domino, fan (see Fig. 1) and the cycles $C_{k}$ of length $k \geq 5$ are not induced subgraphs of $G$.

(3) Every induced subgraph of $G$ contains a leaf or a pair of twins.

(4) For arbitrary vertex $x$ of $G$ and every pair of vertices $v, u \in N^{k}(x)$, that are in the same connected component of the graph $\left\langle V \backslash N^{k-1}(x)\right\rangle$, we have

$$
N(v) \cap N^{k-1}(x)=N(u) \cap N^{k-1}(x) .
$$

(5) (4-point condition)

For any four vertices $u, v, w, x$ of $G$ at least two of the following distance sums are equal:

$$
\operatorname{dist}(u, v)+\operatorname{dist}(w, x) ; \operatorname{dist}(u, w)+\operatorname{dist}(v, x) ; \operatorname{dist}(u, x)+\operatorname{dist}(v, w) .
$$

If the smaller sums are equal, then the largest one exceeds the smaller ones at most by 2.

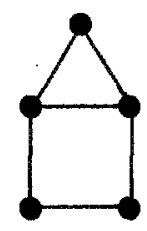

house

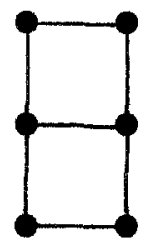

domino

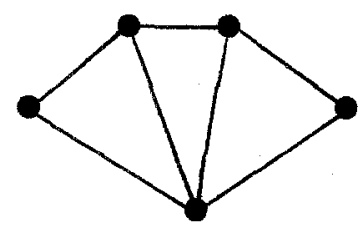

fan

Figure 1: Forbidden induced subgraphs in a distance-hereditary graph.

Proposition 2 ([2],[3]) Let $G$ be a distance-hereditary graph.

(1) Every three pairwise intersecting in $G$ disks have a nonemply intersection. 
(2) For any vertex $v$ of $G$ there is no induced path of length 3 in the graph $\left\langle N^{k}(v)\right\rangle$, i.e. every connected component of $\left\langle N^{k}(v)\right\rangle$ is a cograph.

(3) For any vertex $v$ of $G$ if $u, w$ are vertices in different components of $\left\langle N^{k}(v)>\right.$, then $N(u) \cap N^{k-1}(v)$ and $N(w) \cap N^{k-1}(v)$ are either disjoint, or one of the two sets is contained in the other.

\section{Existence of r-Dominating Cliques}

In this section we give a simple criterion for the existence of an $r$-dominating clique in a given distance-hereditary graph. This criterion is the same as in Helly or in chordal graphs [9].

We say that a subset $M$ of $V$ has an $r$-dominating clique $C$ iff for every vertex $v \in M \operatorname{dist}(v, C) \leq r(v)$ holds, where $\operatorname{dist}(v, C)=\min \{\operatorname{dist}(v, w): w \in C\}$.

Theorem $\left.1{ }^{*}\right)$ Let $G=(V, E)$ be a distance-hereditary graph with $n$-tuple $\left(r\left(v_{1}\right), \ldots, r\left(v_{n}\right)\right)$ of non-negative integers and $M \subseteq V$ be any subset of $V$. Then $M$ has an $r$-dominating clique $C$ if and only if for every pair of vertices $v, u \in M$, the inequality

$$
\operatorname{dist}(v, u) \leq r(v)+r(u)+1
$$

holds. Moreover such a clique $C$ can be determined within time $O(|M| \cdot|E|)$.

Proof: " $\Longrightarrow$ " is obvious.

$" \Longleftarrow "$ : Assume that $v_{1}, \ldots, v_{n}$ is an ordering of $V$ such that $M$ consist of the first $|M|$ vertices of this ordering. Let $i$ be the largest index such that there is a clique $C$ with property $\operatorname{dist}\left(v_{j}, C\right) \leq r\left(v_{j}\right)$ for all vertices $v_{j} \in M, 1 \leq j \leq i$. If $i<|M|$ then for $v_{i+1} \in M \operatorname{dist}\left(v_{i+1}, C\right) \geq r\left(v_{i+1}\right)+1$ holds.

Let $N^{k}(v, u)=N^{k}(v) \cap N^{d i s t(u, v)-k}(u)$. Consider the set

$$
X=\bigcup_{x \in C} N^{r\left(v_{i+1}\right)+1}\left(v_{i+1}, x\right) .
$$

Since $X \subseteq N^{r\left(v_{i+1}\right)+1}\left(v_{i+1}\right)$ and $C \subseteq V \backslash N^{r\left(v_{i+1}\right)}\left(v_{i+1}\right)$ vertices of $X$ belong to the same connected component of graph $\left\langle V \backslash N^{r\left(v_{i+1}\right)}\left(v_{i+1}\right)\right\rangle$. By Proposition 1 (4) for any two vertices $v^{\prime}, v^{\prime \prime} \in X$ we have

$$
N\left(v^{\prime}\right) \cap N^{r\left(v_{i+1}\right)}\left(v_{i+1}\right)=N\left(v^{\prime \prime}\right) \cap N^{r\left(v_{i+1}\right)}\left(v_{i+1}\right) .
$$

So, there is at least one vertex $u_{i+1}$ such that $X \subset N\left(u_{i+1}\right)$ and $\operatorname{dist}\left(u_{i+1}, v_{i+1}\right)=$ $r\left(u_{i+1}\right)$.

Now consider a new clique $C^{\prime} \cup\left\{u_{i+1}\right\}$, where $C^{\prime}$ is a maximal (w.r.t. set inclusion) clique of graph $\langle X\rangle$, containing the clique $C \cap X$ if $C \cap X \neq \emptyset$. Next we show that $C^{\prime} \cup\left\{u_{i+1}\right\}$ is an $r$-dominating clique for the vertices $v_{1}, \ldots, v_{i}, v_{i+1}$. This will be a contradiction to the maximality of $i$.

Pick an arbitrary index $j, j \leq i$, and let $x_{j}$ be a vertex from $C$ with $\operatorname{dist}\left(x_{j}, v_{j}\right) \leq$ $r\left(v_{j}\right)$. The following cases may arise.

*) This theorem was independently proven by F.NrKoLAI [Algorithmische und strukturelle Aspekte distanz-erblicher Graphen, Dissertation Universität Duisburg, 1994] 
Case (1). Either $\operatorname{dist}\left(x_{j}, v_{i+1}\right)=r\left(v_{i+1}\right)+2$ and $\operatorname{dist}\left(x_{j}, v_{j}\right)<r\left(v_{j}\right)$

$$
\text { or } \operatorname{dist}\left(x_{j}, v_{i+1}\right)>r\left(v_{i+1}\right)+2 \text {. }
$$

Since $\operatorname{dist}\left(v_{i+1}, v_{j}\right) \leq r\left(v_{i+1}\right)+r\left(v_{j}\right)+1$ the disks

$$
D^{r\left(v_{i+1}\right)+2}\left(v_{i+1}\right), D^{r\left(v_{j}\right)-1}\left(v_{j}\right), D^{\text {dist }\left(x_{j}, v_{i+1}\right)-r\left(v_{i+1}\right)-2}\left(x_{j}\right)
$$

are pairwise intersecting. (Note that the disks $D^{p}(v)$ and $D^{q}(u)$ intersect if and only if $\operatorname{dist}(v, u) \leq p+q$.$) By Proposition 2$ (1) there exists a vertex $z$ with properties $\operatorname{dist}\left(v_{i+1}, z\right)=r\left(v_{i+1}\right)+2, \operatorname{dist}\left(x_{j}, z\right)+\operatorname{dist}\left(z, v_{i+1}\right)=\operatorname{dist}\left(x_{j}, v_{i+1}\right)$ and $\operatorname{dist}\left(v_{j}, z\right) \leq$ $r\left(v_{j}\right)-1$. We claim that $z$ is adjacent to all vertices from $X$.

Let $u$ be an arbitrary vertex of $X$. Hence $u \in N^{r\left(v_{i+1}\right)+1}\left(v_{i+1}, x\right)$ for some vertex $x \in C$. Evidently, vertex $u^{\prime} \in N^{1}(u, x)$ and vertex $z$ lie in the same connected component of graph $\left\langle V \backslash N^{r\left(v_{i+1}\right)+1}\left(v_{i+1}\right)\right\rangle$. Applying once more Proposition 1 (4) we obtain that $N\left(u^{\prime}\right) \cap N^{r\left(v_{i+1}\right)+1}\left(v_{i+1}\right)=N(z) \cap N^{r\left(v_{i+1}\right)+1}\left(v_{i+1}\right)$, i. e. vertices $u$ and $z$ are adjacent. Thus we have $X \subset N(z)$ and so $\operatorname{dist}\left(v_{j}, C^{\prime}\right) \leq \operatorname{dist}\left(v_{j}, z\right)+1 \leq r\left(v_{j}\right)$.

Case (2). $\operatorname{dist}\left(x_{j}, v_{i+1}\right)=r\left(v_{i+1}\right)+2$ and $\operatorname{dist}\left(x_{j}, v_{j}\right)=r\left(v_{j}\right)$.

Now consider the disks

$$
D^{r\left(v_{i+1}\right)+1}\left(v_{i+1}\right), D^{r\left(v_{j}\right)}\left(v_{j}\right), D^{1}\left(x_{j}\right) \text {. }
$$

They are pairwise intersecting. Again by Proposition 2 (1) there is a vertex $z$ such that $\operatorname{dist}\left(v_{i+1}, z\right)=r\left(v_{i+1}\right)+1, \operatorname{dist}\left(x_{j}, z\right)+\operatorname{dist}\left(z, v_{i+1}\right)=\operatorname{dist}\left(x_{j}, v_{i+1}\right)$ and $\operatorname{dist}\left(v_{j}, z\right) \leq$ $r\left(v_{j}\right)$. Since $z \in X$ vertices $z$ and $u_{i+1}$ are adjacent. Also as in case (1) we can show that $X \subset N\left(x_{j}\right)$.

If $\operatorname{dist}\left(z, v_{j}\right)<r\left(v_{j}\right)$ then we immediately obtain $\operatorname{dist}\left(v_{j}, u_{i+1}\right) \leq r\left(v_{j}\right)$. So, assume that $\operatorname{dist}\left(x_{j}, v_{j}\right)=\operatorname{dist}\left(z, v_{j}\right)=r\left(v_{j}\right)$ and $\operatorname{dist}\left(v_{j}, C^{\prime} \cup\left\{u_{i+1}\right\}\right) \geq r\left(v_{j}\right)+1$. Since $X \subset N\left(x_{j}\right)$ in fact we have $C^{\prime} \cup\left\{u_{i+1}\right\} \subseteq N^{r\left(v_{j}\right)+1}\left(v_{j}\right)$. By Proposition 1 (4) for any two vertices $u^{\prime}, u^{\prime \prime} \in C^{\prime} \cup\left\{u_{i+1}\right\} N\left(u^{\prime}\right) \cap N^{r\left(v_{j}\right)}\left(v_{j}\right)=N\left(u^{\prime \prime}\right) \cap N^{r\left(v_{j}\right)}\left(v_{j}\right)$ holds. That is, $C^{\prime} \cup\left\{u_{i+1}\right\} \subset N(z)$. This implies a contradiction with maximality of clique $C^{\prime} \subset X$.

Case (3). $\operatorname{dist}\left(x_{j}, v_{i+1}\right)=r\left(v_{i+1}\right)+1$.

In this case the inequality $\operatorname{dist}\left(v_{j}, C^{\prime}\right) \leq r\left(v_{j}\right)$ follows from the choice of clique $C^{\prime}$ ( $C^{\prime}$ contains all vertices from $\left.C \cap X\right)$.

Time bound.

$i$-th step: The set $X$ can be determined within time $O(|E|)$. A maximal clique $C^{\prime} \subseteq X$ and vertex $u_{i+1}$ can be found also within time $O(|E|)$.

There are at most $|M|$ such steps, and each step requires at most $O(|E|)$ time.

The eccentricity of vertex $v \in V$ is $e(v)=\max \{\operatorname{dist}(v, u): u \in V\}$. The diameter $\operatorname{diam}(G)$ of $G$ is the maximum eccentricity, while the radius radi $(G)$ of $G$ is the minimum eccentricity of vertices of $G$.

Corollary 1 Let $r(v)=k$ for all $v \in V$. Then a distance-hereditary graph $G$ has a $k$-dominating clique if and only if $\operatorname{diam}(G) \leq 2 k+1$.

Corollary 2 Let $G$ be a distance-hereditary graph. Then $G$ has a dominating clique if and only if $\operatorname{diam}(G) \leq 3$.

Corollary 3 ([16]) For every distance-hereditary graph $G$ we have $\operatorname{diam}(G) \geq 2 \operatorname{radi}(G)-2$.

For the case of chordal graphs Corollary 2 initially occurs in [15]. 


\section{Minimum r-Dominating Cliques}

Let $\gamma_{r}(G)=\min \{|D|: D$ an r-dominating set in $G\}, \gamma_{r-c l i q u e}(G)=\min \{|D|:$ $D$ an r-dominating clique in $G\}$. The problem dual to the minimum $r$-dominating clique problem is: for a given graph $G$ with $n$-tuple $\left(r\left(v_{1}\right), \ldots, r\left(v_{n}\right)\right)$ of nonnegative integers find a maximum cardinality vertex set $P$ such that for all $v, u \in P \operatorname{dist}(u, v)=r(u)+r(v)+1$ holds. We call such a set $P$ a strict $r$-packing set of $G$ and denote by $\pi_{r}(G)$ the cardinality of a maximum strict r-packing set. It is clear that for every graph $G$ which has an $r$-dominating clique

$$
\pi_{r}(G) \leq \gamma_{r}(G) \leq \gamma_{r-\text { clique }}(G)
$$

holds but in general the parameters do not coincide. The next results show the coincidence of some of these parameters for distance-hereditary graphs that have an $r$-dominating clique.

We say that an $r$-dominating clique $C$ is minimal iff for any vertex $v \in C$ the set $C \backslash\{v\}$ is not r-dominating.

Theorem 2 Let $G$ be a distance-hereditary graph which has an $r$-dominating clique for $n$-tuple $\left(r\left(v_{1}\right), \ldots, r\left(v_{n}\right)\right)$ of non-negative integers. If $\gamma_{r-c l i q u e}(G)>1$ then every minimal $r$-dominating clique of $G$ is also a minimum one.

Corollary 4 Let $G$ be a distance-hereditary graph which has an r-dominating clique for the $n$-tuple $\left(r\left(v_{1}\right), \ldots, r\left(v_{n}\right)\right)$ of non-negative integers.

(1) If $C$ is a minimal $r$-dom. clique of $G$ and $|C| \neq 2$ then $\gamma_{r-\text { clique }}(G)=|C|$.

(2) If $\gamma_{r-e l i q u e}(G) \neq 2$ then $\gamma_{r-c l i q u e}(G)=\pi_{r}(G)$.

(3) If $\pi_{r}(G)>1$ then $\gamma_{r-\text { clique }}(G)=\pi_{r}(G)$.

(4) $\gamma_{r}(G)=\gamma_{r-\text { clique }}(G)$.

Corollary 5 For every system of pairwise intersecting disks of a distancehereditary graph $G$ there exists either a single vertex or a pair of adjacent vertices that meet all disks of this system.

Now we are able to present an $O(|V| \cdot|E|)$ algorithm for finding a minimum $r$-dominating clique in distance-hereditary graphs. For a distance-hereditary graph $G=(V, E)$ given together with n-tuple $\left(r\left(v_{1}\right), \ldots, r\left(v_{n}\right)\right)$ first we can decide within time $O(|V| \cdot|E|)$ whether graph $G$ has a single vertex $r$-dominating all other vertices of $G$. If such a vertex does not exist then we can find also within time $O(|V| \cdot|E|)$ (see Theorem 1) an $r$-dominating clique $C$ of $G$ (or decide that such a clique does not exist). Finally we can reduce this clique to a minimal one in time $O(|C| \cdot|E|)$. By Theorem 2 the obtained $r$-dominating clique is a minimum $r$-dominating clique of graph $G$.

Observe that a maximum strict $r$-packing set of a distance-hereditary graph $G$ can be determined also within time $O(|V| \cdot|E|)$ (see Theorem 2).

As we will show in the next section, using the existence of special kinds of vertices (leaves and twins) in distance-hereditary graphs, one can construct a more efficient algorithm for these two problems. 


\section{Algorithm for Finding Minimum r-Domina- ting Cliques}

As we already mentioned, every induced subgraph of distance-hereditary graph contains a leaf or a pair of twins. In the subsequent linear-time algorithm for the minimum $r$-dominating clique problem on distance-hereditary graphs these kinds of vertices turn out to be of importance.

Lemma 1 ([9]) Let $x$ be a leaf in graph $G$ and let $y$ be its neighbor. Let also $G-x=\langle V \backslash\{x\}>$ and $r(x) \geq 1$. A clique $C \subseteq V \backslash\{x\}$ is a minimum $r$ dominating clique of graph $G$ if $\bar{C}$ is a minimum $r^{\prime}$-dominating clique of graph $G-x$ with $r^{\prime}(v)=r(v)$ when $v \neq y$ and $r^{\prime}(y)=\min \{r(y), r(x)-1\}$. In particular, the graph $G$ has an $r$-dominating clique if and only if the graph $G-x$ has an $r^{\prime}$-dominating clique.

Instead of twins next we consider some of their generalizations. A homogeneous set of a graph $G=(V, E)$ is a set of the vertices $A$ such that every vertex in $V \backslash A$ is adjacent to either all or none of the vertices of $A$. A proper homogeneous set is a homogeneous set $A$ such that $|A| \leq|V|-2$. Observe that two vertices are twins if they form a homogeneous set of size 2. Evidently every vertex $v \in V \backslash A$ is equidistant from the vertices of a homogeneous set $A$.

Lemma 2 Let $A \subset V$ be a proper homogeneous set of graph $G$, and $x$ be a vertex of $A$ with $r(x)=\min \{r(y): y \in A\}$. Assume that either $r(x) \geq 2$, or $r(x)=1$ and there exists a vertex $v \in V \backslash A$ with dist $(v, x)>r(v)$. A clique $C \subseteq V \backslash A \cup\{x\}$ is a minimum $r$-dominating clique of graph $G$ if $C$ is a minimum $r$-dominating clique of graph $<V \backslash A \cup\{x\}>$. In particular, the graph $G$ has an $r$-dominating clique if and only if the graph $<V \backslash A \cup\{x\}>$ has an r-dominating clique.

Lemma 3 Let $x$ be a vertex of graph $G$ such that $N(x)$ forms a proper homogeneous set in $G$, and $y$ be a verlex of $N(x)$ with $r(y)=\min \{r(z): z \in N(x)\}$. Assume that $r(x)>r(y)$. A clique $C \subseteq V \backslash\{x\}$ is a minimum r-dominating clique of graph $G$ if $C$ is a minimum $r$-dominating clique of graph $G-x$. In particular, the graph $G$ has an $r$-dominating clique if and only if the graph $G-x$ has an r-dominating clique.

These lemmas will be used in the correctness proof of the subsequent algorithm which has a structure similar to the linear-time recognition algorithm presented in [11] for distance-hereditary graphs.

Algorithm RDC (Find a minimum $r$-dominating clique of a distance-hereditary graph if there is one, and answer NO otherwise)

Input: A distance-hereditary graph $G=(V, E)$ and an $n$-tuple $\left(r\left(v_{1}\right), \ldots, r\left(v_{n}\right)\right)$ of non-negative integers.

Output: A minimum $r$-dominating clique $D C$ of $G$ if there is one and answer NO otherwise. 


\section{begin}

(1) if for all $v \in V r(v)>0$ then

(2) for arbitrary vertex $u \in V$ build its i-neighborhoods $N^{1}(u), N^{2}(u), \ldots, N^{k}(u)$;

(3) for $i=k, k-1, \ldots, 2$ do

(4) find the connected components $A_{1}, A_{2}, \ldots, A_{p}$ of $N^{i}(u) \cap V$;

(5) in each component $A_{j}$ pick a vertex $x_{j}$ such that $r\left(x_{j}\right)=\min \left\{r(y): y \in A_{j}\right\}$;

(6) order the vertices of $X=\left\{x_{1}, x_{2}, \ldots, x_{p}\right\}$ by increasing degree $d^{\prime}\left(x_{j}\right)=\left|N\left(x_{j}\right) \cap N^{i-1}(u)\right|$

(36) stop with output $D C:=\{u\}$

(37) else (now $r(u)=0$ for some $u \in V$ )

outloop:

(38) in the rest of graph $G$ build i-neighborhoods $N^{1}(u), \ldots, N^{k}(u)$ of vertex $u$ with $r(u)=0$

(39) for $i=k, k-1, \ldots, 2$ do

(40) repeat the steps $(4),(5),(6)$; 
(41) for all vertices $x_{j} \in X$ taken by increasing degree $d^{\prime}\left(x_{j}\right)$ do

(51) endfor (now $N[u]=V$ )

(52) put $D C:=\{v \in N[u]: \tau(v)=0\}$;

(53) if $D C$ is no clique then output "there is no $r$-dominating clique in $G$ "

(54) else $D C$ is a minimum $r$-dominating clique of $G$

(55) endif

(56) endif

end

Theorem 3 Algorithm $R D C$ is correct and works in linear time $O(|E|)$.

The following algorithm solves in linear time the maximum strict $r$-packing problem on distance-hereditary graph having an $r$-dominating clique.

Algorithm RSP (Find a maximum strict $r$-packing set of a distance-hereditary graph which has an $r$-dominating clique)

Input: A distance-hereditary graph $G=(V, E)$ and an $n$-tuple $\left(r\left(v_{1}\right), \ldots, r\left(v_{n}\right)\right)$ of non-negative integers.

Output: A maximum strict $r$-packing set $S P$ of $G$.

\section{begin}

(1) by Algorithm $R D C$ find a minimum $r$-dominating clique $D C$ of graph $G$;

(2) if $|D C|=1$ then $S P:=D C$

(3) else $W:=V ; i:=1$;

(4) for all $v \in D C$ do

(5) $\quad \operatorname{num}(v):=1 ; l(v):=0 ; \operatorname{pr}(v):=v ; W:=W \backslash\{v\}$

(6) endfor

(7) for all $v \in V$ with $\operatorname{dist}(v, D C)=1$ do

(8) $\quad \operatorname{num}(v):=|N(v) \cap D C| ; l(v):=1 ; W:=W \backslash\{v\}$;

(9) if num $(v):=1$ then $p r(v):=N(v) \cap D C$ and $g(v):=v$ endif

(10) endfor

(11) while $W \neq \emptyset$ do

(12) for all vertices $x$ with $l(x)=i$ do

for all vertices $y$ from $N(x) \cap W$ do

$$
\operatorname{num}(y):=\operatorname{num}(x) ;(y):=i+1 ; W:=W \backslash\{y\}
$$

if $n u m(y):=1$ then $\operatorname{pr}(y):=\operatorname{pr}(x)$ and $g(y):=g(x)$ endif endfor

endfor 
(18)

(19)

(20) for all $v \in V$ do

(39) endif

end

$$
i:=i+1
$$

enddo endfor

else $(|D C|=2)$

do case

case $r(x)=0$,

case $r(y)=0$;

endif

endcase

endif

endif

if $n u m(v)=1$ and $l(v)=r(v)$ then $p n(p r(v)):=v$ and $p n(g(v)):=v$ endif

if $|D C| \geq 3$ then $S P:=\{p n(u): u \in D C\}$

let $x$ and $y$ be vertices from $D C$;

$S P:=\{x, p n(y)\}$

$S P:=\{p n(x), y\}$

otherwise $(r(y) \geq 1, r(x) \geq 1)$

$X:=\{g(v): n u m(v)=1$ and $l(v)=r(v)$ and $\operatorname{pr}(v)=x\}$;

$Y:=\{g(v): n u m(v)=1$ and $l(v)=r(v)$ and $p r(v)=y\}$;

if (there is a pair of non-adjacent vertices $u \in X$ and $w \in Y$ ) then

$$
S P:=\{p n(u), p n(w)\}
$$

else $S P:=\{x\}$

Theorem 4 Algorithm RSP is correct and works in linear time $O(|E|)$.

\section{Related Problems}

\subsection{Central Vertex, Radius and Diameter}

Recall that a vertex whose eccentricity is equal to $\operatorname{radi}(G)$ is called a central vertex of graph $G$. Denote by $F(v)$ the set of all furthest from $v$ vertices of $G$, i.e. $F(v)=\{u \in V: \operatorname{dist}(v, u)=e(v)\}$.

Lemma 4 For any vertex $v$ of a distance-hereditary graph $G$ and any furthest vertex $u \in F(v)$ we have $e(u) \geq 2 \operatorname{radi}(G)-3$.

Now we present linear-time algorithms for computing a central vertex, a central clique, the radius and diameter of a distance-hereditary graph $G$.

Let $G=(V, E)$ be a distance-hereditary graph. According to lemma 4 the value $k=[(e(u)+1) / 2]$ is an approximation of the radius of $G$, more precisely either $k=\operatorname{radi}(G)$ or $k=\operatorname{radi}(G)-1$. Next we apply the algorithm $R D C$ to graph $G$ with $r(v)=k$ for all $v \in V$. If there exists an $r$-dominating clique in $G$ then any vertex $v$ from a minimum $r$-dominating clique $D C$ is a central vertex of $G$, and $\operatorname{radi}(G)=k$ when $|D C|=1$ and $\operatorname{radi}(G)=k+1$ otherwise. If such a clique does not exist then $\operatorname{radi}(G)=k+1$ and a single vertex of a minimum $r$-dominating clique of $G$ with $r(v)=k+1$ for all $v \in V$ must be central. 
For computing the diameter of $G$ additionally to algorithm $R D C$ we apply the algorithm $R S P$ to graph $G$ with $r(v)=\operatorname{radi}(G)-1$ for all $v \in V$. If there exists an $r$-dominating clique then $\operatorname{diam}(G)=2 \operatorname{radi}(G)-1$ when the maximum strict $r$-packing set $S P$ of $G$ has at least two vertices and $\operatorname{diam}(G)=2 \operatorname{radi}(G)-2$ otherwise. If such a clique does not exist then $\operatorname{diam}(G)=2 \operatorname{radi}(G)$.

The algorithm $R D C$ can be used for finding a central clique of a distancehereditary graph too. The eccentricity $e(C)$ of a clique $C$ is the minimum distance from any vertex to $C$. A clique center of $G$ is a clique with minimum eccentricity which is called the clique radius of $G$ and is denoted by cradi $(G)$. For arbitrary graph $G$ we have $\operatorname{radi}(G) \geq \operatorname{cradi}(G) \geq \operatorname{radi}(G)-1$, because the eccentricity of any clique containing a central vertex of $G$ is at most $\operatorname{radi}(G)$. So, it is sufficient to decide whether $G$ contains an $r$-dominating clique with $r(v)=\operatorname{radi}(G)-1$ for all $v \in V$. Summarizing the results of this subsection we obtain

Theorem 5 The radius, the diameter, a central vertex and a central clique of a distance-hereditary graph can be found in linear time.

\section{2 r-Dominating by Cliques and r-Dominant Cliques}

Since a graph does not necessarily have a dominating clique in [9] we consider the following weaker $r$-domination by cliques problem: Given a graph $G=(V, E)$ with radius function $\left(r\left(v_{1}\right), \ldots, r\left(v_{n}\right)\right)$ of non-negative integers find a minimum number of cliques $C_{1}, \ldots, C_{k}$ such that $\bigcup_{i=1}^{k} C_{i} r$-dominates $G$. Note that for the special case $r\left(v_{i}\right)=0$ for all $i \in\{1, \ldots, n\}$ this is the well-known problem clique partition.

Another problem closely related to that is to find a clique in $G$ which $r$ dominates a maximum number of vertices. We call this the $r$-dominant clique problem. For the special case $r\left(v_{i}\right)=0$ for all $i \in\{1, \ldots, n\}$ this is again a well-known problem namely the maximum clique problem.

It is obvious that these two problems are $N P$-complete. The following results show that for distance-hereditary graphs the problems are solvable in polynomial time.

For a graph $G=(V, E)$ with disks $\mathcal{D}(G)=\left\{D^{k}(v): v \in V\right.$ and $k \geq 0$ a non-negative integer $\}$ let $\Gamma(G)$ be the following graph whose vertices are the disks of $G$ and two disks $D^{p}(v), D^{q}(u)$ are adjacent iff $D^{p+1}(v) \cap D^{q}(u) \neq \emptyset$ (or, equivalently, $D^{p}(v) \cap D^{q+1}(u) \neq 0$ i.e. $\left.0<\operatorname{dist}(v, u) \leq p+q+1\right)$.

Lemma 5 For each distance-hereditary graph $G$ the graph $\Gamma(G)$ is weakly chordal.

This lemma together with Theorem 1 will be used in the following.

Theorem 6 The problem $r$-dominating by cliques is polynomial-time solvable on distance-hereditary graphs.

Theorem 7 The r-dominant clique problem is polynomial-time solvable on distance-hereditary graphs. 


\section{References}

[1] H.-J.Bandelt, A.Henkmann, and F.Nicolai Powers of distance-hereditary graphs, Technical Report SM-DU-220, University Duisburg 1993.

[2] H.-J.BANDELT and H.M.Mulder, Distance-hereditary graphs, J. Comb. Theory Ser.B, 41 (1986) 182-208.

[3] H.-J.Bandelt and H.M.Mulder, Pseudo-modular graphs, Discrete Math., 62 (1986) 245-260.

[4] A.BrandstäDt, Special graph classes - a survey, Technical Report SM-DU-199, University Duisburg 1993.

[5] A. Brandstädt, F.F. Dragan, V.D. Chepoi, and V.I. Voloshin, Dually chordal graphs, Technical Report $S M-D U-225$, University of Duisburg 1993, to appear as extended abstract in the proceedings of WG'93, (ed. J. van Leeuwen), Utrecht, The Netherlands, submitted to SIAM J. Discr. Math.

[6] A.BrandstäDT and D.Kratsch, Domination problems on permutation and other graphs, Theoretical Computer Science, 54 (1987) 181-198.

[7] G.J.Chang , Centers of chordal graphs, Graphs and Combinatorics, 7 (1991) 305-313.

[8] A.D'ATRI and M.MoscarinI, Distance-hereditary graphs, Steiner trees and connected domination, SIAM J. Computing, 17 (1988) 521-538.

[9] F.F.DragaN and A.BrandstäDT, r-Dominating cliques in Helly graphs and chordal graphs, Technical Report SM-DU-228, University Duisburg 1993, Proc. of the 11th STACS, Caen, France, Springer, LNCS 775, 735 - 746, 1994

[10] M.C.Golumbic, Algorithmic Graph Theory and Perfect Graphs, Academic Press, 1980.

[11] P.L.Hammer and F.MafFray, Completely separable graphs, Discrete Appl. Math., 27 (1990) 85-99.

[12] S.C.Hedetniem and R.Laskar, (eds.), Topics on Domination, Annals of Discr. Math. 48, North-Holland, 1991.

[13] E.HoworkA, A characterization of distance-hereditary graphs, Quart. J. Math. Oxford Ser. 2, 28 (1977) 417-420.

[14] D.Kratsch, Finding dominating cliques efficiently in strongly chordal graphs and undirected path graphs, Annals of Discr. Math. 48, Topics on Domination, (S.C.Hedetniemi, R.Laskar, eds.), 225-238.

[15] D.Kratsch, P.Damaschke and A.Lubiw, Dominating cliques in chordal graphs, to appear in Discrete Mathematics.

[16] S.V.Yushmanov and V.D.ChEPoI, A general method of investigation of characteristic of graphs related with eccentricity Math. Problems of Cybernetics, Moscow 31991 217-232 (Russian, English transl.) 